ARTICLE

\title{
Lytic archaeal viruses infect abundant primary producers in Earth's crust
}

Janina Rahlff (1) 1,5,6, Victoria Turzynski ${ }^{1,6}$, Sarah P. Esser (1) ${ }^{1}$, Indra Monsees', Till L. V. Bornemann', Perla Abigail Figueroa-Gonzalez ${ }^{1}$, Frederik Schulz (D) $^{2}$, Tanja Woyke (i) ${ }^{2}$, Andreas Klingl ${ }^{3}$, Cristina Moraru ${ }^{4} \&$ Alexander J. Probst (iD ${ }^{1 凶}$

The continental subsurface houses a major portion of life's abundance and diversity, yet little is known about viruses infecting microbes that reside there. Here, we use a combination of metagenomics and virus-targeted direct-geneFISH (virusFISH) to show that highly abundant carbon-fixing organisms of the uncultivated genus Candidatus Altiarchaeum are frequent targets of previously unrecognized viruses in the deep subsurface. Analysis of CRISPR spacer matches display resistances of $\mathrm{Ca}$. Altiarchaea against eight predicted viral clades, which show genomic relatedness across continents but little similarity to previously identified viruses. Based on metagenomic information, we tag and image a putatively viral genome rich in protospacers using fluorescence microscopy. VirusFISH reveals a lytic lifestyle of the respective virus and challenges previous predictions that lysogeny prevails as the dominant viral lifestyle in the subsurface. CRISPR development over time and imaging of 18 samples from one subsurface ecosystem suggest a sophisticated interplay of viral diversification and adapting CRISPR-mediated resistances of $\mathrm{Ca}$. Altiarchaeum. We conclude that infections of primary producers with lytic viruses followed by cell lysis potentially jump-start heterotrophic carbon cycling in these subsurface ecosystems.

\footnotetext{
${ }^{1}$ Department of Chemistry, Environmental Microbiology and Biotechnology (EMB), Group for Aquatic Microbial Ecology, University of Duisburg-Essen, Essen, Germany. ${ }^{2}$ DOE Joint Genome Institute, Berkeley, CA, USA. ${ }^{3}$ Plant Development \& Electron Microscopy, Biocenter LMU Munich, PlaneggMartinsried, Germany. ${ }^{4}$ Institute for Chemistry and Biology of the Marine Environment (ICBM), Carl-von-Ossietzky-University Oldenburg, 
E arth's continental subsurface harbors $2-6 \times 10^{29}$ prokaryotic cells $^{1,2}$, which represent a major component of life's diversity on our planet ${ }^{3,4}$. Among these organisms are some of the most enigmatic archaea, including Aigarchaeota, Asgard archaea, Altiarchaeota, and members of the DPANN radiation ${ }^{5-8}$. Although the ecology and diversity of subsurface microorganisms has been under investigation in several studies, the fundamental question relating to how microbial diversity and composition in the deep subsurface change with virus infection remains mostly unanswered. Viruses have long been recognized as major drivers of microbial diversification ${ }^{9}$, yet little is known about their lifestyle, activity, and impact on oligotrophic subsurface ecosystems. Recent evidence demonstrated high numbers of virus-cell ratios in marine subsurface sediments ${ }^{10}$, suggesting ongoing viral proliferation in the deep biosphere. In oceanic surface sediments below $1000 \mathrm{~m}$ depth, virus-mediated lysis of archaea was estimated to be a major contributor to carbon release thus affecting global biogeochemical cycles ${ }^{11}$. In addition, pronounced morphological diversity of bacteriophages with presumably lytic representatives has been found in granitic groundwater of up to $450 \mathrm{~m}$ depth $^{12}$, and might be the result of recombination events, horizontal gene transfer, and lysogeny known to shape microbial communities of the subsurface ${ }^{13}$. The recent recovery of two novel bacteriophage genera with lytic genes from groundwater highlights the potential of subsurface environments for being huge reservoirs of previously unknown viruses ${ }^{14}$. Furthermore, a study on predominant Halanaerobium spp. from anthropogenic subsurface communities (hydraulically fractured wells) suggested long-term host-virus dynamics, extensive viral predation, and adaptive host immunity based on clustered regularly interspaced short palindromic repeats (CRISPR) spacer to protospacer matches ${ }^{15}$. CRISPR systems function as defense mechanisms for bacteria and archaea against mobile genetic elements (MGEs), including viruses ${ }^{16}$. The CRISPR locus is usually flanked by cas genes and interspaced by short variable DNA sequences termed spacers ${ }^{16}$ previously acquired from invading MGEs. The diversification of CRISPR-Cas immunity in the host over geographical distances and time due to preceding viral infections and protospacer mutations has been well-documented, e.g., for Sulfolobus islandicus ${ }^{17}$.

Oligotrophic anaerobic subsurface environments can be populated by a variety of different microorganisms, some of them belonging to the phylum Altiarchaeota ${ }^{7,18}$. In fact, these organisms can reach high abundances in their ecosystems with up to $70 \%$ of the total community in the aquifer or with up to $95 \%$ within the biofilm (BF) they form ${ }^{19,20}$. Members of the genus Candidatus Altiarchaeum - the best-studied representative being $\mathrm{Ca}$. A. hamiconexum ${ }^{7}$-occur in anoxic subsurface environments around the globe 21,22 and fix carbon via the reductive acetyl-CoA (Wood-Ljungdahl) pathway ${ }^{7}$. In certain ecosystems, $\mathrm{Ca}$. Altiarchaea form nearly pure BFs, which are kept together by filamentous cell surface appendages called hami (singular: hamus) ${ }^{23}$. Studies to date demonstrated symbiotic relationships of Ca. Altiarchaea with bacterial partners Thiothrix sp. ${ }^{24}$ and Sulfuricurvum sp. ${ }^{25}$, but also a co-occurrence with the episymbiont $\mathrm{Ca}$. Huberiarchaeum crystalense, belonging to the DPANN clade, has been recently reported ${ }^{18,26}$. A single transmission electron micrograph and the presence of CRISPR systems led to speculations on the existence of $\mathrm{Ca}$. Altiarchaeum viruses in the subsurface 27 . However, mesophilic archaeal viruses from the deep terrestrial subsurface remain highly enigmatic, despite the fact that mesophilic archaeal genomes contain more MGEs than their thermophilic counterparts ${ }^{28}$. The knowledge gap on archaeal viruses is fostered by a lack of their genome entries in public databases $^{29}$, missing marker genes for viruses ${ }^{30}$ and a bias toward viruses related to economical, medical, or biotechnological activities $^{31}$. In addition, only $\sim 150$ archaeal viruses have been isolated and described to date ${ }^{32}$. Recent exhaustive metagenomic surveys aided the discovery of novel archaeal viruses ${ }^{33}$ from multiple ecosystems, including the ocean ${ }^{34,35}$, hot springs $^{36-38}$, and soils 39,40 , and eventually allowed targeting and visualization of an uncultivated virus based on its genome ${ }^{41}$. More recently another correlative phageFISH imaging approach was used to visualize bacteriophages within sponge tissue ${ }^{42}$.

Due to their worldwide distribution and high abundance as the main primary producer in certain continental subsurface ecosystems, $\mathrm{Ca}$. Altiarchaea represent the ideal model genus for studying viruses and their infection mechanisms of mesophilic microorganisms in the subsurface. This is especially relevant because the extent to which lytic infections occur in the continental subsurface is unknown, and lysogeny is assumed to be the predominant viral strategy ${ }^{43}$. In this work, we use metagenomics to predict viruses that infect $\mathrm{Ca}$. Altiarchaea in subsurface ecosystems at four different sites across three continents (Europe, Asia, North America). Using virus-targeted direct-geneFISH (virusFISH), we visualize and characterize the most abundant putative virus from a sulfidic spring in Bavaria, Germany, providing novel insights into the lytic lifestyle of an uncultivated virus, whose genome shows little homologies with sequences in public databases and carries no viral hallmark genes. Our analyses further demonstrate the diversification of CRISPR systems of $\mathrm{Ca}$. Altiarchaea along with a decline in virus abundances over 6 years. We conclude that the kill-the-winner theorem can play an important role in evolutionary and ecosystem processes of the deep biosphere.

\section{Results}

Globally distributed $\mathrm{Ca}$. Altiarchaea have complex CRISPR systems with conserved direct repeat (DR) sequences. Screening of $16 \mathrm{~S}$ ribosomal RNA (rRNA) datasets and metagenomes within $\mathrm{IMG}^{44}$ confirmed a global distribution of organisms belonging to the phylum Altiarchaeota (Fig. 1). We performed metagenomic analyses of four terrestrial subsurface ecosystems ranging from 37 to $352 \mathrm{~m}$ below ground that showed high abundance of the genus Ca. Altiarchaeum (Supplementary Table 1), previously also termed Alti-1 ${ }^{21}$. These ecosystems included (i) an anoxic aquifer accessible through an artesian well (Mühlbacher Schwefelquelle, Isling, MSI ${ }^{25}$ sampled in 2012 and 2018, (ii) a high- $\mathrm{CO}_{2}$ geyser (Geyser Andernach, GA) ${ }^{45}$ both located in Germany, (iii) a sulfidic spring in the US (Alpena County Library Fountain, $\mathrm{ACLF})^{46}$, and (iv) a deep underground laboratory in Japan (Horonobe Underground Research Laboratory, HURL) at 140 and $250 \mathrm{~m} \mathrm{depth}{ }^{22}$. All eight genomes of $\mathrm{Ca}$. Altiarchaeum (Supplementary Data 1) carried genetic information for type I-B CRISPR-Cas immunity including proteins Cas5, Cas7, and Cas8a. Although other Cas proteins were also present in the assembly but remained unbinned, we were able to confirm the presence of Cas3 (Supplementary Data 1) and Cas6 $^{7}$ on scaffolds with the taxonomic annotation of Altiarchaea for the MSI site. Proteins of a type III CRISPR-Cas immunity were found at the ACLF, HURL, and MSI site, including repeat associated mysterious proteins (Cmr) of types III-B and III-C and Csm proteins of the type III-A system (Supplementary Data 1). Confidence in binning CRISPR arrays and assigning DR sequences to $\mathrm{Ca}$. Altiarchaea arose from the 16-fold to 146-fold higher abundance of these organisms (and their CRISPR arrays) in the ecosystems than other microbes (Supplementary Fig. 17,22,46). In addition, two versions of a CRISPR DR sequence assigned to $\mathrm{Ca}$. Altiarchaea were highly conserved across these ecosystems (Supplementary Fig. 2, Supplementary Data 1). DR sequence 1 occurred in all four ecosystems, whereas DR sequence 2 was only found at the HURL and 


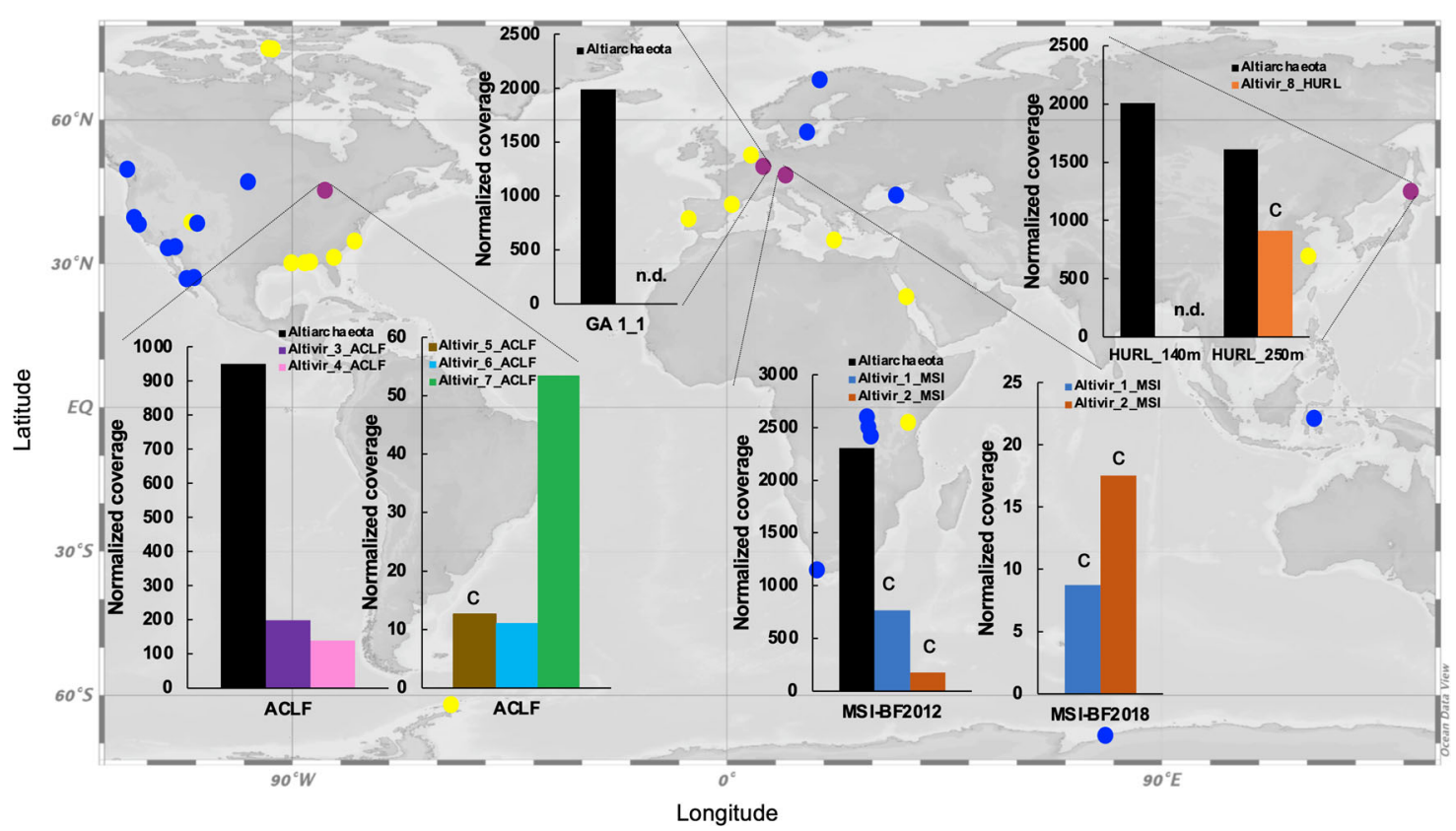

Fig. 1 Global distribution of abundant Altiarchaeota (group Alti-1) and their predicted viruses in Altiarchaeota hot spots. Distribution analysis is based on $16 \mathrm{~S}$ rRNA gene sequencing (yellow dots) and the detection of hamus genes in metagenomes from IMG (blue and purple dots). Purple dots correspond to the four investigated sites of this study. Normalized host and virus coverage are given for the four subsurface habitats: Alpena County Library Fountain (ACLF, Michigan, USA), Horonobe Underground Research Laboratory (HURL, Japan), Mühlbacher Schwefelquelle, Isling (MSI, Germany), and Geyser Andernach (GA, Germany). Percent relative abundance of dominant Altiarchaeota compared to other community members is shown in Supplementary Table 1. Only Altivir_1_MSI and Altivir_2_MSI obtained from biofilm (BF) samples are shown. The letter C indicates circular genomes. n.d. none detected. World map has been generated using Ocean Data View v.5.3.0117. Color explanation of bars representing normalized coverage: orange-red = Altivir_1_MSI (virus), blue = Altivir_2_MSI, purple $=$ Altivir_3_ACLF, rose $=$ Altivir_4_ACLF, brown = Altivir_5_ACLF, light blue $=$ Altivir_6_ACLF, green $=$ Altivir_7_ACLF, and black $=$ Altiarchaeota (host).

the ACLF site (Supplementary Data 1). While all DR sequences from the four sites were previously unknown in the CRISPRmap database, DR sequence 1 in orientation 1 and DR sequence 2 in both orientations structurally resembled motif 13 and 12 of the database, respectively. All four sequences form thermodynamically favorable secondary structures and carry an AAA(N) motif (Supplementary Fig. 2), indicating that both strands of the CRISPR array could theoretically be transcribed.

Eight novel viral clades with genome relatedness across continents show infection histories with Altiarchaeota. Using matches of Altiarchaeota spacers to protospacers, we were able to identify 13 predicted viral genomes (termed $\mathrm{C} a$. Altiarchaeum virus Altivir_\#, here further referred to as Altivir_\# for short) in three out of the four sampling sites (Supplementary Table 2). No viruses targeted by $\mathrm{Ca}$. Altiarchaea spacers could be predicted for GA and the HURL sites at $140 \mathrm{~m}$ depth. Only two out of the 13 predicted viral genomes, i.e., the 20.8-kb long Altivir_2_MSI_BF_2012 and the 22.6-kb long Altivir_8_HURL, had hits in the Virus Orthologous Groups Database (VOGDB, Supplementary Table 2), carried viral hallmark genes, and were circular, prompting us to classify them as viruses. The others were designated as putative viruses according to our classification scheme (Supplementary Fig. 3). All 13 (putative) viruses were categorized as lytic viruses according to VirSorter ${ }^{47}$. Four predicted viruses were circular and thus complete in their genome sequence (Fig. 1, Supplementary Table 2). The 13 viral genomes formed eight monophyletic clades based on VICTOR analysis (Fig. 2), representing potentially eight individual genera (VICTOR threshold for genus was $15.8 \%$ nucleotide based intergenomic similarity). Viral genomes in the Altivir_1_MSI and
Altivir_2_MSI clades were recovered in all sampled time points from the MSI site (Supplementary Table 2), both in the cellular and virus enriched fractions. The Altivir_2_MSI genomes recovered from the virus enriched fraction (MSI_<0.1 $\mu \mathrm{m} \_2018$ ) were fragmented and thus excluded from further analysis. Intergenomic pairwise similarities for the four Altivir_1_MSI varied between 99.3 and $99.7 \%$ (Supplementary Fig. 4), and this clade thus represens a single viral species (the threshold for species demarcation was 95\% similarity). The three Altivir_2_MSI genomes had similarities between 87.0 and $96.1 \%$ and represented two viral species (Supplementary Fig. 4). Using vConTACT, Altivir_1_MSI formed a cluster with Altivir_6_ACLF, a putative virus from ACLF, with whom it shared five protein clusters (Fig. 2, Supplementary Fig. 5). This relatedness between viruses from highly distant subsurface ecosystems was further supported by the VICTOR analysis, which placed them in the same monophyletic clade (Fig. 2). All remaining viruses apart from Altivir_1_MSI, Altivir_2_MSI and Altivir_6_ACLF were designated by vConTACT as unclustered singletons. Only one protein cluster was shared between Altivir_2_MSI, Altivir_4_ACLF, and Altivir_8_HURL, and no protein clusters between the remaining three viruses, indicating that all these viruses are distant from each other (Fig. 2). In total, these eight viral genera were affiliated to seven vConTACT viral clusters (Altivir_1_MSI/ Altivir_6_ACLF grouped together), which were unrelated with previously published viral genomes in the RefSeq94 database according to vConTACT criteria (Supplementary Fig. 5). However, based on the phylogenetic analysis of the DNA polymerase B (for detailed annotations of viral proteins please see below; Supplementary Fig. 6), Altivir_2_MSI is likely related to Tectiviridae, a dsDNA virus family in the Varidnaviria realm. The presence of a capsid portal protein in Altivir_4_ACLF 
a

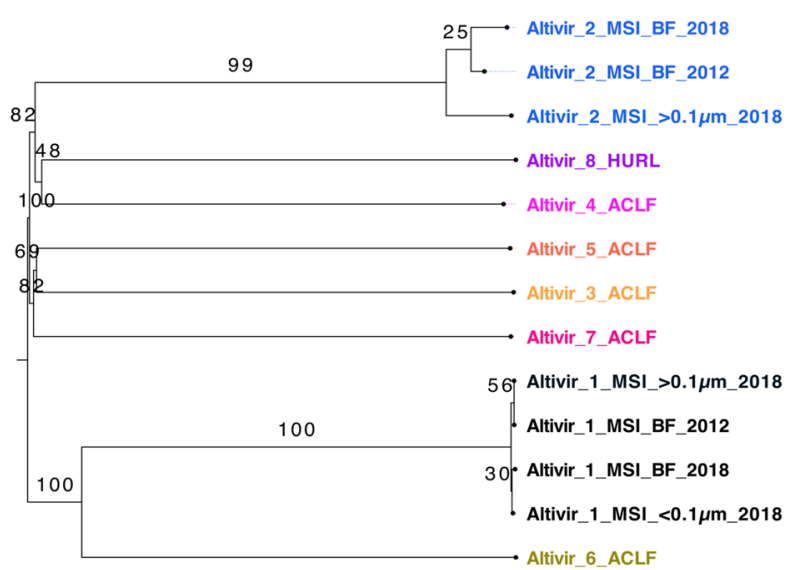

0

0.1

0.2

0.3

0.4

0.5 b

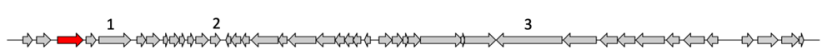

Fig. 2 Phylogenomic genome-BLAST distance phylogeny (GBDP) tree of Ca. Altiarchaeum viruses and viral proteins clusters. a The tree was inferred using the distance formula D0 yielding average support of $69 \%$. The numbers above branches are GBDP pseudo-bootstrap support values from 100 replications. The branch lengths of the resulting VICTOR ${ }^{94}$ trees are scaled in terms of the respective distance formula used. The tree shows that the eight predicted viral genomes were assigned to the same family, to eight different genera, and ten species (which is the result of having multiple genomes included for Altivir_1_MSI and Altivir_2_MSI). b Protein clustering across all viruses revealed that Altivir_1_MSI_>0.1 $\mu$ m_2018 and Altivir_6_ACLF, originating from different continents, have five protein clusters (2-6) in common. Altivir_2_MSI_BF_2012, Altivir_4_ACLF, and Altivir_8_HURL shared only one protein cluster (1). Open reading frames of the viral genomes were predicted using Prodigal v.2.6.3 $3^{74}$ and further translated with the R package seginr v.3.6.-1. Colors indicate shared protein clusters between genomes and numbers show nonhypothetical consensus annotations according to Supplementary Data 3. Color code for viral genomes: Altivir_1_MSI = black, Altivir_2_MSI = blue, Altivir_3_ACLF = yellow, Altivir_4_ACLF = pink, Altivir_5_ACLF = orange, Altivir_6_ACLF = olive-green, Altivir_7_ACLF = pink-red, and Altivir_8_HURL = purple.

(Supplementary Fig. 7), a major tail tube protein in Altivir_7_ACLF (Supplementary Fig. 8), and a terminase as well as a portal protein in Altivir_8_HURL (Supplementary Fig. 9) indicate that these three viruses belong to the Duplodnaviria realm. Phylogenetic analysis of the aforementioned proteins suggests that Altivir_7_ACLF and Altivir_8_HURL potentially belong to the Caudovirales order. Altivir_4_ACLF is distantly related to eukaryotic viruses from Herpesvirales, also suggesting a relationship with Caudovirales.

Comparing the genomes of Altivir_1 and Altivir_2 individually across the samples from 2012 and 2018, we identified different developments of the two viral genomes. Altivir_2_MSI clade presented gene content variations between the genomes from different samples (Supplementary Fig. 10), which agrees with the fact that it represents two viral species. By contrast, Altivir_1_MSI accumulated multiple single-nucleotide polymorphisms and only represented strain level variations of the same species (Supplementary Fig. 10, Supplementary Data 2).

Host-virus ratios (considering $\mathrm{Ca}$. Altiarchaeum to be the host) based on metagenome read mapping varied greatly (between 1.8 and 301.9; Supplementary Table 2) with the smallest ratios of 1.8 for Altivir_8_HURL, followed by 2.3 for Altivir_1_MSI_>0.1 $\mu \mathrm{m} \_2018,2.8$ for Altivir_2_MSI_>0.1 $\mu \mathrm{m} \_2018$, and 3.0 for Altivir_1_MSI_BF_2012. Abundance of in the planktonic fraction $(>0.1 \mu \mathrm{m})$ suggests high concentrations of $\mathrm{Ca}$. Altiarchaeum BF on the $0.1 \mu \mathrm{m}$ filter membrane during the filtration process. Relative abundance of viruses based on normalized coverage ranged between 9 (Altivir_1_MSI_BF_2018) and 913 (Altivir_8_HURL, Fig. 1, Supplementary Table 2). The genomes of Altivir_2_ MSI_BF_2018 and Altivir_8_HURL carried short CRISPR arrays with one spacer each, but spacers from these mini-CRISPR arrays did not match other viruses in the respective ecosystems.

Several viral proteins could be functionally annotated (see Fig. 2B, Supplementary Table 2, Supplementary Data 3), belonging mostly to the circular Altivir_8_HURL genome. Altivir_8_HURL, bearing 36 genes, encoded for an auxiliary metabolic gene (AMG), namely the phosphoadenosine phosphosulfate reductase (PAPS, CysH) (phmmer, $e$-value: 6.1e-48), which probably facilitates assimilatory sulfate reduction in the host, and is a common AMG of many viruses ${ }^{48}$. Altivir_1_MSI, of particular interest for this study because it was highly abundant in the MSI_BF-2012 metagenome, recruited many spacer hits and only 2 out of its 14 proteins could be annotated with enough certainty (Fig. 2, Supplementary Table 2, Supplementary Data 3). These included a nuclease and a geranylgeranyl transferase. In sum, only $11.9 \%$ of the 159 proteins across all Altivir genomes have a putative function assigned rendering the remaining genes of yet unknown function as genetic dark matter (Fig. 2, summary of annotations in Supplementary Table 2).

VirusFISH reveals a lytic lifestyle for Altivir_1_MSI. We selected the in silico predicted Altivir_1_MSI viral clade for visualization by virusFISH, due to its high abundance at the MSI site, and despite the lack of viral hallmark genes. VirusFISH with a probe containing 11 double stranded polynucleotides was successfully implemented to visualize the distribution of the circular genome of Altivir_1_MSI within altiarchaeal BF (Fig. 3A, Supplementary Fig. 11). In contrast to the negative control with a nonmatching probe (Supplementary Fig. 12), our target probes enabled us to detect altiarchaeal cells containing Altivir_1_MSI. Multiple cells were surrounded by halo signals, corresponding to a viral burst ${ }^{49}$ and providing evidence for Altivir_1_MSI being an active virus and lysing $\mathrm{Ca}$. Altiarchaeum cells.

A total of 18,411 altiarchaeal cells and 502 viral infections (colocalization of Altivir_1_MSI and $\mathrm{Ca}$. Altiarchaea signals) across 18 samples/BF flocks were analyzed via fluorescence microscopy and categorized into three main infection stages: (i) 


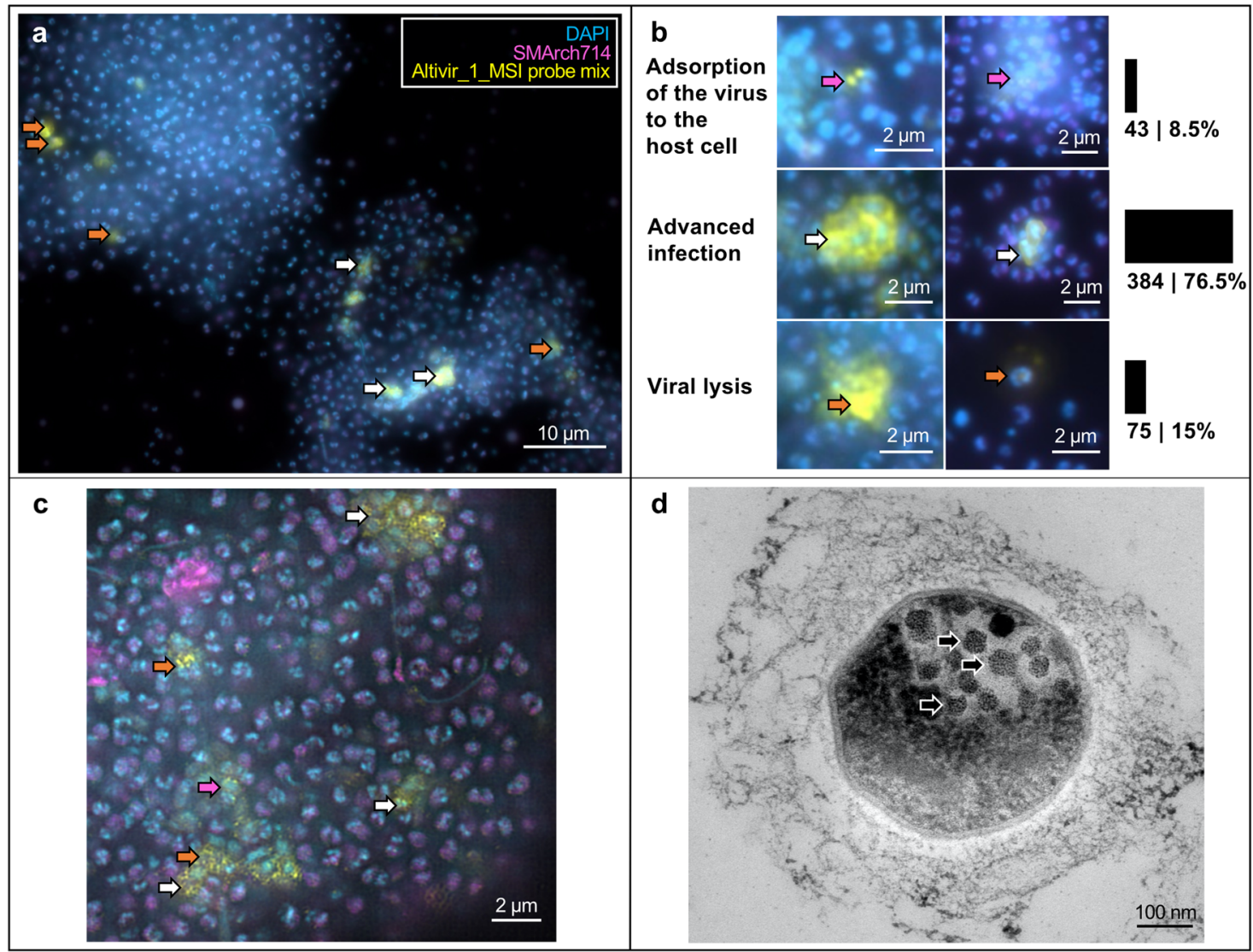

Fig. 3 Visualization and quantification of Ca. Altiarchaeum virus Altivir_1_MSI-infected and noninfected Altiarchaeota biofilm (BF) cells from MSI site. For all virusFISH experiments shown here, an Altivir_1_MSI probe was used for detecting viral infections. BF material was visualized with filter sets for DAPI (blue, cells), ATTO 488 (purple, 16S rRNA signal), and Alexa 594 (yellow, viral genomes), and merged for analysis and display purposes. a VirusFISH displays the interactions between Altiarchaeota cells and their virus shown as yellow dots. For unmerged imaging data, see Supplementary Fig. 11. Scale bar: $10 \mu \mathrm{m}$. b Different infection stages with Altivir_1_MSI. The enumeration performed with a regular epifluorescence microscope was based on 18,411 archaeal cells and categorized into three infection stages. For the categorization we used in total 18 Altiarchaeota biofilms, whereby 17 biofilms were treated with the Altivir_1_MSI probe $(n=17)$ and one biofilm was treated with a Metallosphaera sp. virus probe $(n=1)$. Purple arrows indicate exemplary viruses that attach to host's cell surface, white arrows show advanced infections, and orange arrows bursting cells with free viruses. Scale bars: $2 \mu \mathrm{m}$. c Coupling virusFISH with structured illumination microscopy showed extracellular signals of tiny fluorescently labeled viral particles attaching to

Altiarchaeota's cell surface but also in a free state as presumably released virions $(n=1)$. Scale bar: $2 \mu \mathrm{m}$. $\mathbf{d}$ Transmission electron microscopy revealed intracellular virus-like particles $(n=3)$. Scale bar: $100 \mathrm{~nm}$.

viral adsorption to the host cells (8.5\%), (ii) advanced infection with intracellular virus signals and ring-like signals around the cells (76.5\%), and (iii) cell lysis with bursting cells and release of virions (15\%) (Fig. 3B). Super-resolution microscopy further showed extracellular signals of small fluorescently labeled particles, which we interpret as individual viral particles (Fig. 3C). This observation was further supported by ultrathin sectioning and transmission electron microscopy, which revealed many intracellular virus-like particles associated with $\mathrm{Ca}$. Altiarchaea cells. These particles had an average diameter of $50 \mathrm{~nm}(\mathrm{SD} \pm 7$ $\mathrm{nm}$ ), as measured across 11 host cells (Fig. 3D). The high percentage of cell lysis associated with the virus signals along with the high abundance of the virus in the planktonic and viral fraction suggests a lytic lifestyle for Altivir_1_MSI.

Spatio-temporal heterogeneity of predicted infections and CRISPR-Cas mediated immunity of Ca. Altiarchaea. To investigate the development of virus immunities over time, we compared the publicly available metagenome from MSI (taken in 2012) to a newly sequenced BF sample from 2018. We also analyzed the planktonic microbiome $(>0.1 \mu \mathrm{m})$ and a viral fraction $(<0.1 \mu \mathrm{m})$, i.e., after $0.1 \mu \mathrm{m}$ filtration and $\mathrm{FeCl}_{3}$ precipitation. We compared the change in relative abundance (normalized coverages) of Ca. Altiarchaeum, Altivir_1_MSI, Altivir_2_MSI, and Ca. Altiarchaeum CRISPR spacers across these samples (Fig. 4) with $\mathrm{Ca}$. Altiarchaeum being the most dominant microbe in each sample (Supplementary Fig. S1). While the planktonic microbiome showed a tremendous diversity based on rpS3 sequences (238 different organisms, Fig. 4), the diversity was quite restricted with 19 and 17 organisms in MSI_BF_2012 and MSI_BF_2018, respectively. Please note that there is a difference between the numbers of organisms detected in the rank abundance curves (Supplementary Fig. 1) and those reported in Fig. 4 as the latter were normalized to read abundance to ensure comparability.

Both predicted viral genomes (Altivir_1_MSI_BF_2012, Altivir_2_MSI_BF_2012) declined in abundance when comparing the BF sample from 2018 to the sample from 2012, and at the same time the host-virus ratio increased (Fig. 4). While Altivir_1_MSI was more abundant in 2012 (host-virus ratio $=3.2$ ) compared to Altivir_2_MSI relative to its host (host-virus ratio $=12.4$ ), the pattern reversed in 2018 for the BF sample (host-virus ratio= 299.3 compared to 94.1 for Altivir_1_MSI and Altivir_2_MSI, 


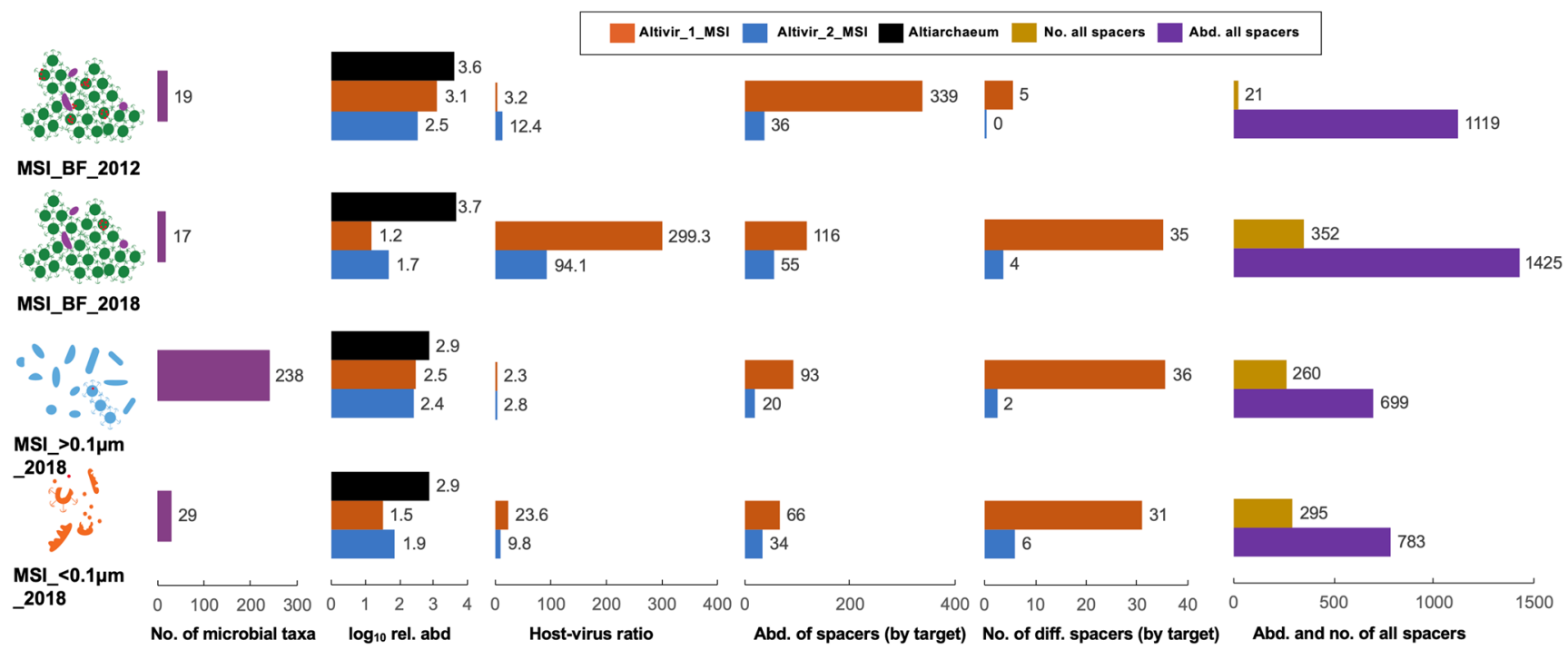

Fig. 4 Development of host-virus and spacer dynamics from 2012 to 2018 based on metagenomics from the Mühlbacher Schwefelquelle, Isling (MSI). Predicted viruses Altivir_1_MSI and Altivir_2_MSI became less abundant from 2012 to 2018. Data are presented for biofilms (BF) from 2012 and 2018 as well as the planktonic fraction $(>0.1 \mu \mathrm{m})$ and the viral fraction $(<0.1 \mu \mathrm{m})$. Considering the BFs, total spacer abundance and numbers increased from 2012 to 2018 while those matching Altivir_1_MSI decreased in abundance but increased in numbers. Number (no.) of microbial taxa refers to the number of different prokaryotes in a sample detected via rpS3 rank abundance curves (normalized by sequencing depth). Host-virus ratio is calculated from host and virus coverage based on read mapping. Abundance and number of different spacers were normalized to minimum relative abundance (rel. abd.) of the host based on read mapping. Color definition of bars: violet = number of microbial taxa, orange-red = features of Altivir_1_MSI, blue = features of Altivir_2_MSI, black $=\log _{10}$ relative abundance of Altiarchaeum, ocher $=$ number of all spacers, and purple $=$ abundance of all spacers.

respectively). Both, the total spacer abundance and the spacer diversity increased from 2012 to 2018 in BF samples, i.e., from 21 to 352 (number of different spacers) including an $\sim 20 \%$ increase in the number of spacer clusters that were singletons in the dataset (Supplementary Fig. 13) and from 1119 to 1425 (abundance of spacers). The abundance of spacers matching the genome of Altivir_1_MSI_BF_2012 decreased from 339 to 116 spacers, whereas it increased from 36 to 55 for Altivir_2_MSI_BF_2012 in BF samples from 2012 to 2018. For both targets the number of different matching spacers increased over time (Supplementary Fig. 14), in line with the development of the total spacers in this ecosystem (Fig. 4). Because planktonic $\mathrm{Ca}$. Altiarchaeum cells (diameter: $0.4-0.6 \mu \mathrm{m}$ ) cannot pass the 0.1 $\mu \mathrm{m}$ pore-size filters, it is more likely that lysed $\mathrm{Ca}$. Altiarchaeum cells ended up in the $<0.1 \mu \mathrm{m}$ fraction in 2018 allowing binning of their population genome including the CRISPR system with low complexity of spacers from this fraction. The MSI_>0.1 $\mu \mathrm{m} \_2018$ fraction and the MSI_<0.1 $\mu \mathrm{m} \_2018$ contained about half the number of total spacers of the MSI_BF_2018 sample, although the number of different spacers displayed less variability. Spacers from these samples hitting the viral targets were often reduced in abundance compared to BF-derived CRISPR spacers (Fig. 4). MSI_BF_2018 had the most unique total spacer clusters (2599, Supplementary Fig. 15A) and also the most unique ones matching Altivir_1_MSI (Supplementary Fig. 15B) and Altivir_2_MSI as displayed as Venn diagrams in Supplementary Fig. 15C. The overlap of spacer clusters between years and samples was rather moderate ( $\leq 52$ spacer clusters in common).

Congruent with the decline in relative abundance of Altivir_1_MSI based on metagenomic analysis, we also observed heterogeneous infections of $\mathrm{BF}$ flocks via imaging. Some $\mathrm{BF}$ showed no infection with Altivir_1_MSI at all (Fig. 5A, Supplementary Fig. 16), which aligns well with the decrease of the virus in the metagenomic data of the BF from 2012 to 2018. By contrast, we observed very few BF flocks that showed an extremely high infection and accumulation of rod-shaped microorganisms (Fig. 5B, Supplementary Figs. 17 and 18). The observed heterogeneity of infections in BF supports the aforementioned heterogeneity related to CRISPR resistances against Altivir_1_MSI with high spacer diversity dominated by singletons.

\section{Discussion}

Although life in the deep subsurface contributes significantly to overall biomass and microbial biodiversity on our planet, its low accessibility leaves host-virus interactions-especially those of uncultivated hosts-highly enigmatic. Detection of novel and uncultivated viruses missing conserved sets of hallmark genes has been an ongoing challenge in viromics ${ }^{50}$. Using a combination of bioinformatics and virusFISH, we were able to visualize infections of Altiarchaeota with a hitherto unknown, presumably lytic virus from a subsurface ecosystem. We expanded the diversity of this virus detected in Germany (MSI) by identifying a distantly related viral genome infecting $\mathrm{Ca}$. Altiarchaea in North America (ACLF site). This suggests genomic relatedness of archaeal viruses over long distances, a phenomenon known for bacteriophages ${ }^{51,52}$. However, genomic relatedness and global distribution of bacteriophage genomes (and maybe also altiarchaeotal viruses) is in stark contrast to viruses infecting Sulfolobus islandicus. Viruses of this thermoacidophilic archaeon displayed a confined geographic distribution based on core gene sequence analysis and CRISPRbased host-virus interactions ${ }^{53,54}$, which demonstrated infections with local viruses for this host, and the geographical structuring of archaeal viral communities was recently extended by investigations of Italian hydrothermal environments ${ }^{55}$.

Altiarchaeota viruses do not represent an exception regarding the many genes that could not be annotated, and thus were classified as unknown ORFs or domains/genes of unknown function. Some proteins such as modification methylases or the phosphoadenosine phosphosulfate reductase were previously also detected on a Methanosarcina and other archaeal virus genomes $^{56,57}$. Altiarchaeota can apparently employ two different CRISPR systems (types I and III) with high similarity of their DR 

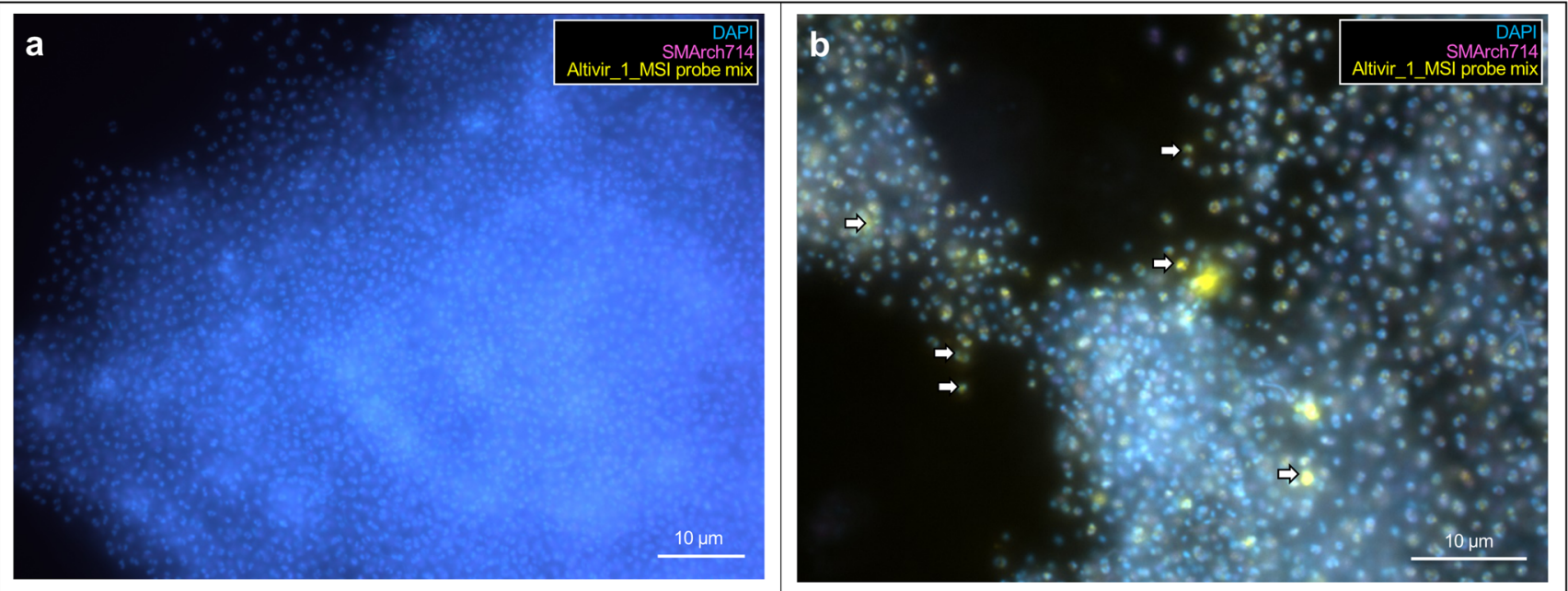

Fig. 5 VirusFISH of two individual Altiarchaeota biofilm (BF) flocks depicting a) a dense BF flock without infections and b) one highly infected BF flock. For all virusFISH experiments, an Altivir_1_MSI probe was used for detecting viral infections. White arrows indicate exemplary virus-Altiarchaeota interactions (advanced infections). BF material was analyzed with filter sets for DAPI (blue, cells), ATTO 488 (purple, 16S rRNA signal), and Alexa 594 (yellow, viral genomes), and then the different fluorescent channels were merged for analysis and display purposes. For unmerged imaging data, see Supplementary Figs. 16 and 17. Scale bars: $10 \mu \mathrm{m}$.

sequences across larger geographic distances; although the reasoning behind two independent CRISPR systems remains unclear, an additional type III system might mediate resistance against plasmids carrying matching protospacers but lacking a protospacer-adjacent motif ${ }^{58}$ or against viruses that overcome type I systems as previously described for Marinomonas mediterrane $a^{59}$ and Sulfolobus islandicus ${ }^{60}$. Indeed, archaeal viruses found ways to interfere with CRISPR type III systems by using anti-CRISPR proteins $s^{61,62}$. However, we could not detect homologs of these proteins in viruses targeting $\mathrm{Ca}$. Altiarchaeum.

Based on the metagenomic data collected in 2012 and 2018, it appears that the host prevailed in the arms race between Altiarchaeaota and the visualized virus (Altivir_1_MSI), whereas the arms race seems to be ongoing as at least parts of the host population still get infected. The number of different spacers matching this virus increased toward 2018 with a prominence of spacer singletons in the metagenome (Supplementary Fig. 13), while the actual abundance of spacers matching Altivir_1_MSI decreased as did the abundance of the viral genome itself. CRISPR spacer diversification might be a successful response to an increasing number of variants in the genome of Altivir_1_MSI (Supplementary Fig. 10) suggesting its mutations. As a trade-off, spacer diversification might allow the host to decrease the total abundance of spacers. We conclude that CRISPR spacers were diversifying to mediate a greater bandwidth of resistances against the virus, which worked in favor for the overall population of $\mathrm{Ca}$. Altiarchaea in this ecosystem. Moreover, the diversity of singleton spacers indicates a very heterogeneous Altiarchaeota population, which might be related to heterogeneous infections of the $\mathrm{BF}$ as visualized via virusFISH.

Our dual approach of coupling metagenomics to fluorescence microscopy enabled us to follow precisely the terrestrial subsurface predator-prey relationship of $\mathrm{Ca}$. Altiarchaeum and one of its viruses. Our data suggest that the novel identified virus is lytic and challenge the current paradigm that lysogeny prevails in the subsurface ${ }^{30,43}$. Instead, our data indicate that the kill-the-winner theorem-lytic viruses targeting abundant ecosystem key players $^{63}$-also strongly applies to subsurface ecosystems. We currently do not know if this statement can be transferred to other subsurface ecosystems that have low cell counts ${ }^{2}$ as viruses might struggle with finding a new host. However, replication measures of bacteria in the ecosystem with the visualized virus suggest that microbial proliferation is similar to other oligotrophic systems ${ }^{45}$. Lytic infections in subsurface microbial hosts might thus launch heterotrophic carbon cycling similar to the viral shunt in the marine environment ${ }^{64}$. In fact, recent lipidomic analyses coupled to mass-balance calculations provide evidence that subsurface environments dominated by Altiarchaeota are completely fueled by these organisms' carbon fixation, transferring organic carbon to heterotrophs in the community ${ }^{65}$. This process might be the basis for microbial loops ${ }^{66}$ as we see the accumulation of rod-shaped microbes around Altiarchaeota when they are lysing due to viral attacks (Supplementary Fig. 18).

Here, we provide underpinning evidence derived from metagenomic datasets of three continents for the frequent viral infection of a globally abundant, autotrophic key player of the subsurface carbon cycle. Using virusFISH for visualization, we show that one virus with a lytic lifestyle is even capable of infecting host cells in a dense BF, which is generally known to provide some protection from viral infection ${ }^{67}$. Subsurface ecosystems such as aquifers remain understudied regarding host-virus dynamics because of limited access, low microbial biomass, and limited cultivation success. Our results presented here provide an experimental proof of concept for an ongoing host-virus arms race in the continental subsurface characterized by constant viral infections and cell lysis of subsurface microbes, followed by their own and their viruses' diversification.

\section{Methods}

Mining public metagenomes for Altiarchaeota. To get an overview of the global distribution of Altiarchaeota, we searched metagenomes in the IMG/M database ${ }^{44}$ (database accessed in July 2018) for Altiarchaeota contigs using DIAMOND BLASTp (v0.9.22) ${ }^{68}$ with the putative hamus subunit (NCBI accession no CEG12198.1) as a query and an $e$-value and length cut-off of $1 \mathrm{e}-10$ and 300 amino acids, respectively. The Altiarchaeota distribution based on 16S rRNA gene sequences was obtained from the SILVA SSU Parc database ${ }^{69}$ based on all 16S rRNA genes classified as Altiarchaeota and for which geographic information was available (July 2018).

To investigate Altiarchaeota-virus relationships, we explored ecosystems where Altiarchaeota comprised the majority of the community. Therefore, metagenomic data from a microbial mat growing in the sulfidic groundwater-fed ACLF (Alpena, MI, USA) were obtained from NCBI's Sequence Read Archive (SRA) repository 46 as were metagenome samples from HURL at 140 and $250 \mathrm{~m}$ depth (HURL, Hokkaido, Japan $)^{22}$. The analysis was complemented with three metagenomic 
datasets from $\mathrm{CO}_{2}$-enriched groundwater erupted from a cold-water Geyser (Andernach, Middle Rhine Valley, Germany) ${ }^{45}$, and the metagenome of a sulfidic spring MSI in Regensburg, Germany ${ }^{7}$. All BioProject, BioSample accessions, and information on sampling of MSI for various experiments are provided in Supplementary Tables 1,3 , and 4 , respectively.

\section{Resampling of MSI for virusFISH and metagenomic sequencing. BF samples} were collected as previously described ${ }^{20}$ from the $36.5 \mathrm{~m}$ deep, cold $\left(\sim 10^{\circ} \mathrm{C}\right)$, sulfidic spring MSI in Regensburg, Germany ( $\mathrm{N} 48^{\circ} 59.142$, E $012^{\circ} 07.636$ ) in January 2019 , for which further geological description has been reported elsewhere ${ }^{70}$. Environmental parameters of the sulfidic spring were extensively investigated previously 25 and remained almost constant over years. In brief, hydrogen sulfide concentration in the spring hole was reported to be $0.85 \mathrm{mg} \mathrm{L}^{-1} 70$, dissolved carbon dioxide at $32 \mathrm{mg} \mathrm{L}^{-125}$, and oxygen at $0.13 \mathrm{mg} \mathrm{L}^{-1}$ and later revised to be beyond detection limit of extremely sensitive probes ${ }^{20,25}$. Chemical parameters of the spring water are presented in Supplementary Table 5.

For virusFISH, BF samples were fixed by addition of formaldehyde $(3 \% \mathrm{v} / \mathrm{v})$ and incubation at room temperature for $1 \mathrm{~h}$. For investigating infection stages, $\mathrm{BF}$ flocks were gently separated from several bigger flocks by using a pipette tip finally yielding 18 smaller flocks (range $170-5870 \mu^{2}$ ). BF flocks for all microscopy experiments were subsequently washed three times in $1 \mathrm{x}$ phosphate buffered saline (PBS, pH 7.4) followed by dehydration via an ethanol gradient $(50 \%, 70 \% \mathrm{v} / \mathrm{v}$, and absolute ethanol, $10 \mathrm{~min}$ each). Samples were stored in absolute ethanol at $-20^{\circ} \mathrm{C}$ until further processing.

Three types of samples were collected for metagenomics: (i) BF flocks; (ii) the planktonic community $(>0.1 \mu \mathrm{m}$ pore-size fraction); and (iii) the viruses and lysed cells $(<0.1 \mu \mathrm{m}$ pore-size fraction). Sampling of Altiarchaeota BF flocks for DNA extraction and metagenomic sequencing from the sulfidic spring (MSI) was performed in October 2018. For sampling the unfiltered planktonic microbial community, $70 \mathrm{~L}$ of groundwater were filtered onto a $0.1 \mu \mathrm{m}$ pore-size PTFE membrane filter (Merck Millipore, Darmstadt, Germany). The flow-through was collected in a sterilized container, and a final concentration of $1 \mathrm{mg} \mathrm{L}^{-1}$ of iron (III) chloride (Carl Roth, Karlsruhe, Germany) was applied for chemical flocculation for $30 \mathrm{~min}^{71}$. Flocculates were filtered onto $5 \times 0.2 \mu \mathrm{m}$ membrane filters $(<0.1 \mu \mathrm{m}$ fraction). DNA was extracted directly from collected BF samples using the RNeasy ${ }^{\circledR}$ PowerBiofilm Kit (Qiagen, Hilden, Germany) using a DNAconform workflow. DNA from the $0.1 \mu \mathrm{m}$ and pooled $0.2 \mu \mathrm{m}$ membrane filters with iron flocculates was extracted using PowerMax Soil DNA Extraction Kit (Qiagen, Hilden, Germany), was precipitated overnight, and cleaned with $70 \%$ ethanol. Shotgun metagenome sequencing was conducted within the Census of Deep Life Sequencing call 2018 and performed using the Illumina HiSeq platform at the Marine Biological Laboratory, Woods Hole, MA, USA.

Detection of viral genomes in metagenomes. Raw shotgun sequencing reads were trimmed and quality-filtered using bbduk (https://github.com/BioInfoTools/ BBMap/blob/master/sh/bbduk.sh) and Sickle v.1.3372. Read assembly was conducted by using metaSPADes v. $3.10^{73}$ unless stated otherwise. Scaffolds $<1 \mathrm{~kb}$ length were excluded from further analysis. Genes were predicted using prodigal v.2.6.3 $3^{74}$ (meta mode) and functional annotations were determined by using DIAMOND v.0.9.968 against UniRef100 (February 2018) ) $^{75}$. Public Altiarchaeota genomes were retrieved from databases ${ }^{45}$ and further cleaned or rebinned using \% GC content, coverage distribution, and taxonomy information ${ }^{76}$, which was necessary for all genomes except for Altiarchaeota from MSI_BF_2012 (Supplementary Table 1). Viral scaffolds $>3 \mathrm{~kb}$ were identified by applying a combination of tools as presented in Supplementary Fig. 3A. Predicted viruses were classified into viruses and putative viruses according to the classification system presented in Supplementary Fig. 3B. Viral scaffolds were subsequently checked for miniCRISPR arrays using default settings of CRISPRCasFinder ${ }^{77}$ as some archaeal viruses can bear mini-CRISPR arrays with 1-2 spacers having likely a role in interviral conflicts ${ }^{78,79}$.

CRISPR-Cas analysis of Altiarchaeota genomes. Cas genes and DR sequences were identified in binned Altiarchaeota genomes via CRISPRCasFinder ${ }^{77}$ and genes were additionally confirmed via searches against UniRef $100^{75}$. CRISPR DR sequences were tested for formation of secondary structures using RNAfold ${ }^{80}$ and checked against the CRISPRmap database (v2.1.3-2014) ${ }^{81}$ for formation of known motifs. The consensus DR sequence was used in both possible orientations to extract host-specific spacers from raw reads by using MetaCRAST ${ }^{82}$ with Cd-hit v.4. $6^{83}$ clustering at $99 \%$ identity. Spacers were filtered for minimum and maximum lengths of 20 and 60 nucleotides, respectively. Only spacers that were present on a read that contained at least one complete DR sequence with an exact match to the template were considered. Finally, spacers were further clustered with Cd-hit ${ }^{83}$ at $99 \%$ identity and matched to viral protospacers on the compiled output of viral identification tools using the BLASTn-short algorithm with a $80 \%$ similarity threshold. For comparing spacer dynamics (total abundance, diversity, and matches to Altivir_1_MSI and Altivir_2_MSI genomes) of 2012 and 2018 samples from MSI, all spacers of the four respective MSI samples were clustered with Cdhit ${ }^{83}$ at $99 \%$ identity and representative sequences of each cluster were matched to representative Altivir_1_MSI and Altivir_2_MSI genomes from the BF of 2012
(Supplementary Table 2). All data arising from spacer counts were normalized by genome abundance of the respective Altiarchaeota genome.

Functional annotation of proteins and clustering of viral genomes. Coding sequences of predicted viral scaffolds were identified using prodigal (meta mode) $)^{74}$

Furthermore, the proteins predicted by prodigal were grouped into clusters based on BLASTp similarity ${ }^{84}$ and then functionally annotated by searching in several sequence databases and then manually curating and consolidating the results. The databases searched were the following: (i) the NR database (virus only section, https://blast.ncbi.nlm.nih.gov/) from NCBI was searched using BLASTp and DELTA-BLAST (if no BLASTp results were found, ${ }^{85}$ ); (ii) the prokaryotic Viruses Orthologous Groups ${ }^{86}$ was searched using HHblits v.3.3.0 (results kept only if the probability was $\geq 70 \%)^{87}$; (iii) the $\operatorname{VOGDB}^{88}$ (vog93, April 2019) was searched using HMMER with an $e$-value cut-off of $10^{-5}$; (iv) the InterPro database $82.0^{89}$ was searched using InterProScan integrated in Geneious Prime 202090; (v) UniRef100 (Feb. 2018) ${ }^{75}$ was searched using DIAMOND ${ }^{68}$ with an $e$-value of $10^{-5}$, and (vi) PDB_mmCIFC70_4_Feb, Pfam-A v.32.0, NCBI_Conserved_Domains_v3.16, and TIGRFAMs v15.0 database were searched using HHpred $^{91,92}$ with an $e$-value cut-off of $10^{-6}$. For the two most abundant predicted $\mathrm{Ca}$. Altiarchaeum viruses Altivir_1_MSI and Altivir_8_HURL, we additionally applied DELTA-BLAST ${ }^{85}$ searches against NCBI's nonredundant protein sequences (nr) and phmmer ${ }^{93}$ against reference proteomes.

Predicted viral scaffolds carrying multiple hits against existing bacterial genomes in UniRef $100^{75}$ and no viral hallmark genes or carrying extensive CRISPR arrays (detected as false positives) were removed from further analyses.

Clustering of entire viral genomes on nucleic acid level was performed with VICTOR $^{94}$ (https://ggdc.dsmz.de/victor.php) using the genome-BLAST distance phylogeny method ${ }^{95}$ with distance formula $\mathrm{d} 0$ and OPTSIL clustering ${ }^{96}$. A separate virus clustering was performed using vConTACT v.0.9.11 $1^{97,98}$ applying the database "ProkaryoticViralRefSeq94"99 and visualization of the viral network was performed in Cytoscape version 3.7.2100. Intergenomic similarities between viral genomes and the corresponding heatmap were calculated using VIRIDIC accessible through http://viridic.icbm.de with default settings ${ }^{101}$. In order to further investigate the genetic relationship between the $\mathrm{Ca}$. Altiarchaeum viruses Altivir_1-Altivir_8, proteins of all viral genomes were clustered by first performing an all against all BLASTp with an $e$-value cut-off of $10^{-5}$ and a bitscore threshold of 50 . Then, the results were loaded into the mcl program with the parameters -1 --abc. The genomic maps were plotted using the genoPlotR v.0.8.9102 package of the $\mathrm{R}$ programming environment v.3.5.2 ${ }^{103}$.

Single-gene phylogenetic trees. Phylogenetic trees were constructed for viral hallmark proteins identified in different $\mathrm{Ca}$. Altiarchaeum viruses: (i) DNA polymerase B from Altivir_2_MSI; (ii) capsid portal protein from Altivir_4_ACLF; (iii) major tail tube protein from Altivir_7_ACLF, and (iv) terminase protein from Altivir_8_HURL. For each phylogenetic tree, the following workflow was performed. The respective viral hallmark protein was used as BLASTp query to search for related proteins in the viral section of the NR BLAST database from NCBI (https://blast.ncbi.nlm.nih.gov/). If no results were found with BLASTp (capsid protein from Altivir_4_ACLF, major tail tube protein from Altivir_7_ACLF), then DELTA-BLAST was used to search for distantly related proteins. Only hits with an $e$-value smaller than 0.0005 were kept. The selected proteins, including the query, were aligned using the Constraint-based Multiple Alignment Tool (COBALT ${ }^{104}$ ), using the online tool (https://www.ncbi.nlm.nih.gov/tools/cobalt/re_cobalt.cgi). The COBALT alignment was imported in Geneious Prime $2020^{90}$ for refinement using MUSCLE v.3.8.425 ${ }^{105}$ and end trimmed. The tree for the Altivir_2_MSI was constructed using FastTree v.2.1.12 ${ }^{106}$ with the "Whelan and Goldman 20012" and "Optimize the Gamma20 likelihood" parameters. The trees for Altivir_4_ACLF, Altivir_7_ACLF and Altivir_8_HURL were calculated using the webserver phylogeny.fr. Here, the alignment was first curated with Gblocks" 107 (option "Do not allow many contiguous nonconserved positions"). The tree was reconstructed using PhyML ${ }^{108}$ with the substitution model "WAG for protein" and the approximate likelihood-ratio test "SH-like" for calculating the branch support statistics. All trees were exported as ".newick" format, visualized with FigTree v.1.4.3 ${ }^{109}$, rooted at the midpoint, and further annotated in Inkscape v.1.0.2 (https://inkscape.org/).

Relative abundance of viruses and hosts. Abundance of hosts and viruses was determined via read mapping against respective genomes using Bowtie $2^{110}$ in sensitive mode followed by mismatch filtering for host genomes ( $2 \%$, depending on read length). Abundances were normalized to the total number of base pairs (bp) sequenced of each sample scaling to the sample with lowest counts, which is herein referred to as relative abundance or normalized coverage. Rank abundance curves were built based on the abundance of scaffolds carrying ribosomal protein S3 $(r p S 3)$ gene sequences predicted in metagenomic assemblies after running prodigal (meta mode) ${ }^{74}$ and annotation ${ }^{68}$ against UniRef $100^{75}$. For normalized rank abundance curves based on sequencing depth for the ecosystem MSI, we only considered $r p S 3$ gene sequences with a coverage $>2.4$ after normalization as this value represents the lowest coverage of any assembled $r p S 3$ gene sequence in the MSI dataset. 
Design, synthesis, and chemical labeling of gene probes. To target Altivir_1_MSI, an $8.9 \mathrm{~kb}$ putative viral genome recovered from the MSI_BF_2012 metagenomic dataset, 11 dsDNA polynucleotide probes of 300 nucleotides length were designed using genePROBER (gene-prober.icbm.de/). In addition, a single $300 \mathrm{bp}$ fragment of a Metallosphaera turreted icosahedral virus strain MTIV1 (NCBI accession no. MF443783.1) was designed as a negative control probe, because the Metallosphaera sp. virus was not detected in the metagenome of MSI_BF_2012. Sequences for all probes are given in Supplementary Tables 6 and 7.

All probes were chemically synthesized by IDT (Integrated DNA Technologies, San Jose, CA, USA) as gBlocks ${ }^{\oplus}$ Gene Fragments (500 ng per polynucleotide), reconstituted in $5 \mathrm{mM}$ Tris- $\mathrm{HCl}$ pH 8.0, $1 \mathrm{mM}$ EDTA pH 8.0, and then labeled with the ULYSIS ${ }^{\text {rx }}$ Alexa Fluor ${ }^{\text {ra }} 594$ nucleic acid labeling kit (Thermo Fisher Scientific, MA, USA) ${ }^{111}$. Two micrograms of either an equimolar mixture of the 11 Altivir_1_MSI polynucleotides or of the single negative control polynucleotide were used in a single labeling reaction and then purified using NucAway Spin Columns (Thermo Fisher Scientific, USA). Before being used as probes for virustargeted direct-geneFISH (virusFISH), the labeled probes were measured spectrophotometrically using a NanoDrop ${ }^{\text {Tw }}$ (Thermo Fisher Scientific, USA). The calculated labeling efficiency was 9.16 and 16.6 dyes per base for Altivir_1_MSI probe and negative control probe, respectively.

\section{VirusFISH of MSI BFs.}

VirusFISH was performed according to the direct-geneFISH protocol ${ }^{112}$, with the modifications detailed further. In brief, Altiarchaeota BF, dehydrated in an ethanol series, was carefully placed in the middle of a press-to-seal silicone isolator (SigmaAldrich Chemie GmbH, Taufkirchen, Germany) mounted on Superfrost ${ }^{\circledast}$ Plus slides (Electron Microscopy Sciences, Hatfield, USA), and subsequently air-dried. Because Altiarchaeota lack the typical archaeal S-layer as outer sheath ${ }^{113}$ and hence are more prone to membrane disintegration, no extra permeabilization step was required. Different formamide concentrations $(20,30$, and $50 \%)$ were tested to exclude false positive hybridization signals. In the final assay, $20 \%$ of formamide was used in the hybridization buffer that contained 5x SSC buffer (saline sodium citrate, $\mathrm{pH} 7.0$ ), $20 \%$ (w/v) dextran sulfate, $20 \mathrm{mM}$ EDTA, $0.25 \mathrm{mg} \mathrm{mL}^{-1}$ sheared salmon sperm DNA, $0.25 \mathrm{mg} \mathrm{mL}^{-1}$ yeast RNA, 1x blocking reagent, $0.1 \%(\mathrm{v} / \mathrm{v})$ sodium dodecyl sulfate, and nuclease-free water. The final $\mathrm{NaCl}$ concentration of $0.225 \mathrm{M}$ in the washing buffer corresponded to the $20 \%$ formamide in the hybridization buffer. As rRNA probes, the dual-Atto488-labeled probes NON338 (5'ACTCCTACGGGAGGCAGC-3' $)^{114}$ and a SM1-Euryarchaeon-specific probe SMARCH714 (5'-GCCTTCGCCCAGATGGTC- $\left.3^{\prime}\right)^{115}$ were used. A volume of 45 $\mu \mathrm{L}$ hybridization mixture was used, with a final gene probe concentration of $330 \mathrm{pg}$ $\mu \mathrm{L}^{-1}\left(30 \mathrm{pg} \mu \mathrm{L}^{-1}\right.$ for each polynucleotide) and rRNA probe final concentration of $1 \mathrm{pmol} \mu \mathrm{L}^{-1}$. In the hybridization chamber, $30 \mathrm{ml}$ of formamide-water solution was added to keep a humid atmosphere. The denaturation and hybridization times were $30 \mathrm{~min}$ and $3 \mathrm{~h}$, respectively. The post hybridization washing buffer contained $20 \mathrm{mM}$ Tris- $\mathrm{HCl}$ ( $\mathrm{pH} 8.0$ ), $5 \mathrm{mM}$ EDTA ( $\mathrm{pH} \mathrm{8.0),} \mathrm{nuclease-free} \mathrm{water,} 0.01 \%$ SDS, and $0.225 \mathrm{M} \mathrm{NaCl}$. A second washing step with 1x PBS (pH 7.4) for 20 min was performed. Then, the slides were transferred for $1 \mathrm{~min}$ into molecular grade water and quickly rinsed in absolute ethanol. For staining, we used $15 \mu \mathrm{L}$ of a mixture of 4',6-diamidin-2-phenylindole $\left(4 \mu \mathrm{g} \mathrm{mL}^{-1}\right)$ in SlowFade Gold Antifade Mounting medium (both from Thermo Fisher Scientific, Waltham, MA, USA). All solutions and buffers for virusFISH experiments were prepared with molecular grade water (Carl Roth, Karlsruhe, Germany). For the experiment with individual flocks, 17 out of 18 flocks were treated with the Altivir_1_MSI probe and one flock with the negative control probe.

The BF material was examined and imaged with an Axio Imager M2m epifluorescence microscope equipped with an AxioCam MRm and a Zen 2 Pro software (version 2.0.0.0) (Carl Zeiss Microscopy GmbH, Jena, Germany). Channel mode visualization was performed by using the $110 \times / 1.3$ oil objective EC-Plan NEOFLUAR (Carl Zeiss Microscopy GmbH) and three different filter sets from Carl Zeiss: 49 DAPI for visualizing Altiarchaeota cells, $64 \mathrm{HE}$ mPlum for the detection of viral infections, and 09 for achieving 16S rRNA signals. Enumeration of cells and viral signals was performed manually. Viral signals were categorized into three major groups, i.e., viral adsorption on host cells, advanced infections, and viral bursts.

Structured illumination microscopy. For structured illumination microscopy, the whole virusFISH protocol was carried out on a BF flock mounted on a cover slip (thickness no. 1.5H, Paul Marienfeld GmbH \& Co. KG, Lauda-Königshofen, Germany). Staining was conducted without adding DAPI to the mounting medium. Samples were analyzed using an inverted epifluorescence microscope (Zeiss ELYRA PS.1) equipped with an $a$-Plan-Apochromat $100 \times / 1.46$ oil DIC M27 Elyra objective, F Set 77 He filter, and a Zen 2.3 SP1 FP3 black edition software (version 14.0.22.201), all obtained from the manufacturer Carl Zeiss Microscopy GmbH, Germany.

Transmission electron microscopy. BF flocks from MSI were prefixed in glutaraldehyde (Carl Roth, Karlsruhe, Germany) to a final concentration of $2.5 \%(\mathrm{v} / \mathrm{v})$ and physically fixed via high-pressure freezing followed by freeze substitution, which was carried out with $0.2 \%(\mathrm{w} / \mathrm{v})$ osmium tetroxide, $0.25 \%(\mathrm{w} / \mathrm{v})$ uranyl acetate, and $9.3 \%(\mathrm{v} / \mathrm{v})$ water in acetone $\mathrm{e}^{116}$. After embedding in Epon resin and polymerization for $72 \mathrm{~h}$, the samples were ultrathin sectioned and poststained with $1 \%$ lead citrate for $2 \mathrm{~min}$. Transmission electron microscopy was carried out on a Zeiss EM 912 (Zeiss, Oberkochen, Germany) with an integrated OMEGA-filter at $80 \mathrm{kV}$ in the zero-loss mode. Imaging was done using a $2 \mathrm{k} \times 2 \mathrm{k}$ pixel slow-scan CCD camera (TRS Tröndle Restlichtverstärkersysteme, Moorenweis, Germany) and an ImageSP software (version 1.2.9.77) (×64) (SysProg, Minsk, Belarus).

Reporting summary. Further information on research design is available in the Nature Research Reporting Summary linked to this article.

\section{Data availability}

Sequencing data generated and analyzed during this study are available at NCBI's SRA and GenBank with accession codes as listed in Supplementary Table 1. Metagenomic reads are available from NCBI Bioprojects \#PRJNA628506, Biosamples \#SAMN14733005-07, Run \#SRR11614986-88 (MSI_2018), Bioproject \#PRJNA321556, Biosample \#SAMN04999996-97, Run \#SRR3546456-57 (HURL), Bioproject \#PRJNA627655 (GA), Biosample \#SAMN14680028-30, Run \#SRR11600161-63 (GA), Bioproject \#PRJNA340050, Biosample \#SAMN05661201, Run \#SRR4293692 (ACLF), as well as from ENA \#PRJNA678866, Biosample \#SAMEA2779769, and Run accession \#ERR628383 (MSI_2012). Metagenomic assemblies can be found under the abovementioned Bioprojects/samples for MSI_2018 and GA. Exceptions represented MSI_2012, which was deposited under Bioproject \#PRJNA678866, Biosample \#SAMN16815598, HURL assemblies under Bioproject \#PRJNA730881, Biosample \#SAMN04999996-7, and the ACLF assembly under Bioproject \#PRJNA730879 and Biosample \#SAMN05661201. Run accessions for these assemblies are

JAEMOC000000000-JAEMOL000000000. Ca. Altiarchaeota genomes were deposited at GenBank under Bioproject \#PRJNA628506, MAG Biosample \#SAMN18220766, Run \#JAGTWS000000000 (MSI_2018), Bioproject \#PRJNA627655, MAG Biosamples \#SAMN18220852-54, Run \#JAGTWP000000000-JAGTWR000000000 (GA), Bioproject \#PRJNA726854, MAG Biosample \#SAMN18221259, Run \#JAGWDR000000000 (ACLF), Bioproject \#PRJNA726852, MAG Biosample \#SAMN18220774-75, Run

\#JAGWDQ000000000 and \#JAGWDP000000000 (HURL), and at ENA under \#CCXY01000000 (MSI_2012). Viral genomes of Ca. Altiarchaeum virus Altivir_1 and 2 can be found under GenBank accessions \#MW522970 and \#MW522971, respectively. Ca. Altiarchaeum virus Altivir_3-8 genomes are available in the Third-Party Annotation Section of the DDBJ/ENA/GenBank databases under the accession numbers \#BK059157162. Viral genomes of $\mathrm{Ca}$. Altiarchaeum virus Altivir_3-8 can also be accessed via the scaffold accession numbers as given in Supplementary Table 4. Viral genomes can also be found at https://github.com/ProbstLab/viromics/tree/master/viruses/Altivir. Microscopy data can be obtained from the corresponding author upon reasonable request.

\section{Code availability}

Our tool Endmatcher for identification of circular sequences from metagenomes is available under https://github.com/ProbstLab/viromics/tree/master/Endmatcher.

Received: 5 August 2020; Accepted: 5 July 2021; Published online: 30 July 2021

\section{References}

1. Flemming, H. C. \& Wuertz, S. Bacteria and archaea on Earth and their abundance in biofilms. Nat. Rev. Microbiol. 17, 247-260 (2019).

2. Magnabosco, C. et al. The biomass and biodiversity of the continental subsurface. Nat. Geosci. 11, 707-717 (2018).

3. Anantharaman, K. et al. Thousands of microbial genomes shed light on interconnected biogeochemical processes in an aquifer system. Nat. Commun. 7, 13219 (2016)

4. Hug, L. A. et al. A new view of the tree of life. Nat. Microbiol. 1, 16048 (2016)

5. Castelle, C. J. et al. Genomic expansion of domain archaea highlights roles for organisms from new phyla in anaerobic carbon cycling. Curr. Biol. 25, 690-701 (2015).

6. Nunoura, T. et al. Insights into the evolution of Archaea and eukaryotic protein modifier systems revealed by the genome of a novel archaeal group. Nucleic Acids Res. 39, 3204-3223 (2011).

7. Probst, A. J. et al. Biology of a widespread uncultivated archaeon that contributes to carbon fixation in the subsurface. Nat. Commun. 5, 5497 (2014).

8. Zaremba-Niedzwiedzka, K. et al. Asgard archaea illuminate the origin of eukaryotic cellular complexity. Nature 541, 353-358 (2017).

9. Weinbauer, M. G. \& Rassoulzadegan, F. Are viruses driving microbial diversification and diversity? Environ. Microbiol. 6, 1-11 (2004).

10. Engelhardt, T., Kallmeyer, J., Cypionka, H. \& Engelen, B. High virus-to-cell ratios indicate ongoing production of viruses in deep subsurface sediments. ISME J. 8, 1503-1509 (2014). 
11. Danovaro, R. et al. Virus-mediated archaeal hecatomb in the deep seafloor. Sci. Adv. 2, e1600492 (2016).

12. Kyle, J. E., Eydal, H. S., Ferris, F. G. \& Pedersen, K. Viruses in granitic groundwater from 69 to $450 \mathrm{~m}$ depth of the Äspö hard rock laboratory, Sweden. ISME J. 2, 571-574 (2008).

13. Labonté, J. M. et al. Single cell genomics indicates horizontal gene transfer and viral infections in a deep subsurface Firmicutes population. Front. Microbiol. 6, 349 (2015).

14. Hylling, O. et al. Two novel bacteriophage genera from a groundwater reservoir highlight subsurface environments as underexplored biotopes in bacteriophage ecology. Sci. Rep. 10, 11879 (2020).

15. Daly, R. A. et al. Viruses control dominant bacteria colonizing the terrestrial deep biosphere after hydraulic fracturing. Nat. Microbiol. 4, 352-361 (2019).

16. Horvath, P. \& Barrangou, R. CRISPR/Cas, the immune system of bacteria and archaea. Science 327, 167-170 (2010).

17. Pauly, M. D., Bautista, M. A., Black, J. A. \& Whitaker, R. J. Diversified local CRISPR-Cas immunity to viruses of Sulfolobus islandicus. Philos. Trans. $R$. Soc. Lond. B Biol. Sci. 374, 20180093 (2019).

18. Probst, A. J. et al. Differential depth distribution of microbial function and putative symbionts through sediment-hosted aquifers in the deep terrestrial subsurface. Nat. Microbiol. 3, 328-336 (2018).

19. Henneberger, R., Moissl, C., Amann, T., Rudolph, C. \& Huber, R. New insights into the lifestyle of the cold-loving SM1 euryarchaeon: natural growth as a monospecies biofilm in the subsurface. Appl. Environ. Microbiol. 72, 192-199 (2006).

20. Probst, A. J. et al. Tackling the minority: sulfate-reducing bacteria in an archaea-dominated subsurface biofilm. ISME J. 7, 635-651 (2013).

21. Bird, J. T., Baker, B. J., Probst, A. J., Podar, M. \& Lloyd, K. G. Culture independent genomic comparisons reveal environmental adaptations for Altiarchaeales. Front. Microbiol. 7, 1221 (2016).

22. Hernsdorf, A. W. et al. Potential for microbial $\mathrm{H}_{2}$ and metal transformations associated with novel bacteria and archaea in deep terrestrial subsurface sediments. ISME J. 11, 1915-1929 (2017).

23. Moissl, C., Rachel, R., Briegel, A., Engelhardt, H. \& Huber, R. The unique structure of archaeal 'hami', highly complex cell appendages with nanograppling hooks. Mol. Microbiol. 56, 361-370 (2005).

24. Rudolph, C., Wanner, G. \& Huber, R. Natural communities of novel archaea and bacteria growing in cold sulfurous springs with a string-of-pearls-like morphology. Appl. Environ. Microbiol. 67, 2336-2344 (2001).

25. Rudolph, C., Moissl, C., Henneberger, R. \& Huber, R. Ecology and microbial structures of archaeal/bacterial strings-of-pearls communities and archaeal relatives thriving in cold sulfidic springs. FEMS Microbiol. Ecol. 50, 1-11 (2004).

26. Schwank, K. et al. An archaeal symbiont-host association from the deep terrestrial subsurface. ISME J. 13, 2135-2139 (2019).

27. Probst, A. J. \& Moissl-Eichinger, C. "Altiarchaeales": uncultivated archaea from the subsurface. Life 5, 1381-1395 (2015).

28. Makarova, K. S. et al. Dark matter in archaeal genomes: a rich source of novel mobile elements, defense systems and secretory complexes. Extremophiles 18, 877-893 (2014).

29. Vik, D. R. et al. Putative archaeal viruses from the mesopelagic ocean. PeerJ 5, e3428 (2017)

30. Anderson, R. E., Brazelton, W. J. \& Baross, J. A. The deep viriosphere: assessing the viral impact on microbial community dynamics in the deep subsurface. Carbon Earth 75, 649-675 (2013).

31. Rodrigues, R. A. L. et al. An anthropocentric view of the virosphere-host relationship. Front. Microbiol. 8, 1673 (2017).

32. Munson-McGee, J. H., Snyder, J. C. \& Young, M. J. Archaeal viruses from high-temperature environments. Genes 9, 128 (2018).

33. Paez-Espino, D. et al. Uncovering Earth's virome. Nature 536, 425-430 (2016).

34. Philosof, A. et al. Novel abundant oceanic viruses of uncultured marine group II Euryarchaeota. Curr. Biol. 27, 1362-1368 (2017).

35. Ahlgren, N. A., Fuchsman, C. A., Rocap, G. \& Fuhrman, J. A. Discovery of several novel, widespread, and ecologically distinct marine Thaumarchaeota viruses that encode $a m o C$ nitrification genes. ISME J. 13, 618-631 (2019).

36. Gudbergsdottir, S. R., Menzel, P., Krogh, A., Young, M. \& Peng, X. Novel viral genomes identified from six metagenomes reveal wide distribution of archaeal viruses and high viral diversity in terrestrial hot springs. Environ. Microbiol. 18, 863-874 (2016).

37. Munson-McGee, J. H., Rooney, C. \& Young, M. J. An uncultivated virus infecting a nanoarchaeal parasite in the hot springs of Yellowstone National Park. J. Virol. 94, e01213-19 (2020).

38. Zablocki, O., van Zyl, L. J., Kirby, B. \& Trindade, M. Diversity of dsDNA viruses in a South African hot spring assessed by metagenomics and microscopy. Viruses 9, 348 (2017).

39. Emerson, J. B. et al. Host-linked soil viral ecology along a permafrost thaw gradient. Nat. Microbiol. 3, 870-880 (2018).
40. Trubl, G. et al. Soil viruses are underexplored players in ecosystem carbon processing. mSystems 3, 338103 (2018).

41. Hochstein, R. A., Amenabar, M. J., Munson-McGee, J. H., Boyd, E. S. \& Young M. J. Acidianus tailed spindle virus: a new archaeal large tailed spindle virus discovered by culture-independent methods. J. Virol. 90, 3458-3468 (2016).

42. Jahn, M. T. et al. Lifestyle of sponge symbiont phages by host prediction and correlative microscopy. ISME J. 15, 1-11 (2021).

43. Anderson, R. E., Brazelton, W. J. \& Baross, J. A. Is the genetic landscape of the deep subsurface biosphere affected by viruses? Front. Microbiol. 2, 219 (2011).

44. Chen, I. A. et al. IMG/M v.5.0: an integrated data management and comparative analysis system for microbial genomes and microbiomes. Nucleic Acids Res. 47, D666-D677 (2019).

45. Bornemann, T. L. V. et al. Geological degassing enhances microbial metabolism in the continental subsurface. https://doi.org/10.1101/ 2020.03.07.980714 (2020).

46. Sharrar, A. M. et al. Novel large sulfur bacteria in the metagenomes of groundwater-fed chemosynthetic microbial mats in the Lake Huron Basin. Front. Microbiol. 8, 791 (2017).

47. Roux, S., Enault, F., Hurwitz, B. L. \& Sullivan, M. B. VirSorter: mining viral signal from microbial genomic data. PeerJ 3, e985 (2015).

48. Kieft, K. et al. Virus-associated organosulfur metabolism in human and environmental systems. Cell Reports, in press (2021).

49. Allers, E. et al. Single-cell and population level viral infection dynamics revealed by phageFISH, a method to visualize intracellular and free viruses. Environ. Microbiol. 15, 2306-2318 (2013).

50. Roux, S. et al. Minimum information about an uncultivated virus genome (MIUViG). Nat. Biotechnol. 37, 29-37 (2019).

51. Breitbart, M. \& Rohwer, F. Here a virus, there a virus, everywhere the same virus? Trends Microbiol. 13, 278-284 (2005).

52. Short, C. M. \& Suttle, C. A. Nearly identical bacteriophage structural gene sequences are widely distributed in both marine and freshwater environments. Appl. Environ. Microbiol. 71, 480-486 (2005).

53. Bautista, M. A., Black, J. A., Youngblut, N. D. \& Whitaker, R. J. Differentiation and structure in Sulfolobus islandicus rod-shaped virus populations. Viruses $\mathbf{9}$, 120 (2017).

54. Held, N. L. \& Whitaker, R. J. Viral biogeography revealed by signatures in Sulfolobus islandicus genomes. Environ. Microbiol. 11, 457-466 (2009).

55. Baquero, D. P. et al. New virus isolates from Italian hydrothermal environments underscore the biogeographic pattern in archaeal virus communities. ISME J. 14, 1821-1833 (2020).

56. Molnár, J. et al. Identification of a novel archaea virus, detected in hydrocarbon polluted Hungarian and Canadian samples. PLOS ONE 15, e0231864 (2020).

57. Prangishvili, D., Garrett, R. A. \& Koonin, E. V. Evolutionary genomics of archaeal viruses: unique viral genomes in the third domain of life. Virus Res. 117, 52-67 (2006).

58. Deng, L., Garrett, R. A., Shah, S. A., Peng, X. \& She, Q. A novel interference mechanism by a type IIIB CRISPR-Cmr module in Sulfolobus. Mol. Microbiol. 87, 1088-1099 (2013).

59. Silas, S. et al. Type III CRISPR-Cas systems can provide redundancy to counteract viral escape from type I systems. Elife 6, e27601 (2017).

60. Guo, T., Han, W. \& She, Q. Tolerance of Sulfolobus SMV1 virus to the immunity of IA and III-B CRISPR-Cas systems in Sulfolobus islandicus. RNA Biol. 16, 549-556 (2019).

61. Athukoralage, J. S. et al. An anti-CRISPR viral ring nuclease subverts type III CRISPR immunity. Nature 577, 572-575 (2020).

62. Bhoobalan-Chitty, Y., Johansen, T. B., Di Cianni, N. \& Peng, X. Inhibition of type III CRISPR-Cas immunity by an archaeal virus-encoded anti-CRISPR protein. Cell 179, 448-458 e411 (2019).

63. Thingstad, T. F. \& Lignell, R. Theoretical models for the control of bacterial growth rate, abundance, diversity and carbon demand. Aquat. Microbiol. Ecol. 13, 19-27 (1997).

64. Wilhelm, S. W. \& Suttle, C. A. Viruses and nutrient cycles in the sea-viruses play critical roles in the structure and function of aquatic food webs. Bioscience 49, 781-788 (1999).

65. Probst, A. J. et al. Lipid analysis of $\mathrm{CO}_{2}$-rich subsurface aquifers suggests an autotrophy-based deep biosphere with lysolipids enriched in CPR bacteria. ISME J. 14, 1547-1560 (2020).

66. Dong, $\mathrm{X}$. et al. Fermentative spirochaetes mediate necromass recycling in anoxic hydrocarbon-contaminated habitats. ISME J. 12, 2039-2050 (2018).

67. Vidakovic, L., Singh, P. K., Hartmann, R., Nadell, C. D. \& Drescher, K. Dynamic biofilm architecture confers individual and collective mechanisms of viral protection. Nat. Microbiol. 3, 26-31 (2018).

68. Buchfink, B., Xie, C. \& Huson, D. H. Fast and sensitive protein alignment using DIAMOND. Nat. Methods 12, 59-60 (2015).

69. Quast, C. et al. The SILVA ribosomal RNA gene database project: improved data processing and web-based tools. Nucleic Acids Res. 41, D590-D596 (2013). 
70. Probst, A. J. et al. Coupling genetic and chemical microbiome profiling reveals heterogeneity of archaeome and bacteriome in subsurface biofilms that are dominated by the same archaeal species. PLOS ONE 9, e99801 (2014).

71. John, S. G. et al. A simple and efficient method for concentration of ocean viruses by chemical flocculation. Environ. Microbiol. Rep. 3, 195-202 (2011).

72. Joshi, N. \& Fass, J. Sickle: a sliding-window, adaptive, quality-based trimming tool for FastQ files (Version 1.33) [Software]. https://github.com/najoshi/ sickle (2011).

73. Nurk, S., Meleshko, D., Korobeynikov, A. \& Pevzner, P. A. metaSPAdes: a new versatile metagenomic assembler. Genome Res. 27, 824-834 (2017).

74. Hyatt, D. et al. Prodigal: prokaryotic gene recognition and translation initiation site identification. BMC Bioinform. 11, 119 (2010).

75. Suzek, B. E., Huang, H., McGarvey, P., Mazumder, R. \& Wu, C. H. UniRef: comprehensive and non-redundant UniProt reference clusters. Bioinformatics 23, 1282-1288 (2007).

76. Bornemann, T. L. V., Esser, S. P., Stach, T. L., Burg, T. \& Probst, A.J. uBin-a manual refining tool for metagenomic bins designed for educational purposes. https://doi.org/10.1101/2020.07.15.204776 (2020).

77. Couvin, D. et al. CRISPRCasFinder, an update of CRISRFinder, includes a portable version, enhanced performance and integrates search for Cas proteins. Nucleic Acids Res. 46, W246-W251 (2018).

78. Medvedeva, S. et al. Virus-borne mini-CRISPR arrays are involved in interviral conflicts. Nat. Commun. 10, 5204 (2019).

79. Iranzo, J., Faure, G., Wolf, Y. I. \& Koonin, E. V. Game-theoretical modeling of interviral conflicts mediated by mini-CRISPR arrays. Front. Microbiol. 11, 381 (2020).

80. Denman, R. B. Using Rnafold to predict the activity of small catalytic RNAs. Biotechniques 15, 1090-\& (1993).

81. Lange, S. J., Alkhnbashi, O. S., Rose, D., Will, S. \& Backofen, R. CRISPRmap: an automated classification of repeat conservation in prokaryotic adaptive immune systems. Nucleic Acids Res. 41, 8034-8044 (2013).

82. Moller, A. G. \& Liang, C. MetaCRAST: reference-guided extraction of CRISPR spacers from unassembled metagenomes. PeerJ 5, e3788 (2017).

83. Li, W. \& Godzik, A. Cd-hit: a fast program for clustering and comparing large sets of protein or nucleotide sequences. Bioinformatics 22, 1658-1659 (2006).

84. Bischoff, V. et al. Cobaviruses-a new globally distributed phage group infecting Rhodobacteraceae in marine ecosystems. ISME J. 13, 1404-1421 (2019).

85. Boratyn, G. M. et al. Domain enhanced lookup time accelerated BLAST. Biol. Direct 7, 12 (2012)

86. Grazziotin, A. L., Koonin, E. V. \& Kristensen, D. M. Prokaryotic virus orthologous groups (pVOGs): a resource for comparative genomics and protein family annotation. Nucleic Acids Res. 45, D491-D498 (2017).

87. Remmert, M., Biegert, A., Hauser, A. \& Söding, J. HHblits: lightning-fast iterative protein sequence searching by HMM-HMM alignment. Nat. Methods 9, 173-175 (2011).

88. Marz, M. et al. Challenges in RNA virus bioinformatics. Bioinformatics 30, 1793-1799 (2014).

89. Finn, R. D. et al. InterPro in 2017-beyond protein family and domain annotations. Nucleic Acids Res. 45, D190-D199 (2017).

90. Kearse, M. et al. Geneious basic: an integrated and extendable desktop software platform for the organization and analysis of sequence data. Bioinformatics 28, 1647-1649 (2012).

91. Söding, J., Biegert, A. \& Lupas, A. N. The HHpred interactive server for protein homology detection and structure prediction. Nucleic Acids Res. 33, W244-W248 (2005).

92. Zimmermann, L. et al. A completely reimplemented MPI bioinformatics toolkit with a new HHpred server at its core. J. Mol. Biol. 430, 2237-2243 (2018).

93. Potter, S. C. et al. HMMER web server: 2018 update. Nucleic Acids Res. 46, W200-W204 (2018).

94. Meier-Kolthoff, J. P. \& Göker, M. VICTOR: genome-based phylogeny and classification of prokaryotic viruses. Bioinformatics 33, 3396-3404 (2017).

95. Meier-Kolthoff, J. P., Auch, A. F., Klenk, H. P. \& Göker, M. Genome sequence-based species delimitation with confidence intervals and improved distance functions. BMC Bioinform. 14, 60 (2013).

96. Göker, M., Garcia-Blazquez, G., Voglmayr, H., Telleria, M. T. \& Martin, M. P. Molecular taxonomy of phytopathogenic fungi: a case study in Peronospora. PLoS ONE 4, e6319 (2009).

97. Bin Jang, $H$. et al. Taxonomic assignment of uncultivated prokaryotic virus genomes is enabled by gene-sharing networks. Nat. Biotechnol. 37, 632-639 (2019).

98. Bolduc, B. et al. vConTACT: an iVirus tool to classify double-stranded DNA viruses that infect archaea and bacteria. PeerJ 5, e3243 (2017).

99. Brister, J. R., Ako-Adjei, D., Bao, Y. \& Blinkova, O. NCBI viral genomes resource. Nucleic Acids Res. 43, D571-D577 (2015).

100. Shannon, P. et al. Cytoscape: a software environment for integrated models of biomolecular interaction networks. Genome Res. 13, 2498-2504 (2003).
101. Moraru, C., Varsani, A. \& Kropinski, A. M. VIRIDIC-A novel tool to calculate the intergenomic similarities of prokaryote-infecting viruses. Viruses 12, 1268 (2020)

102. Guy, L., Kultima, J. R. \& Andersson, S. G. genoPlotR: comparative gene and genome visualization in R. Bioinformatics 26, 2334-2335 (2010).

103. Team RC. R: a language and environment for statistical computing. ( $\mathrm{R}$ Foundation for Statistical Computing, 2019). https://www.R-project.org/.

104. Papadopoulos, J. S. \& Agarwala, R. COBALT: constraint-based alignment tool for multiple protein sequences. Bioinformatics 23, 1073-1079 (2007).

105. Edgar, R. C. MUSCLE: a multiple sequence alignment method with reduced time and space complexity. BMC Bioinform. 5, 113 (2004).

106. Price, M. N., Dehal, P. S. \& Arkin, A. P. FastTree 2-approximately maximum-likelihood trees for large alignments. PLoS ONE 5, e9490 (2010).

107. Castresana, J. Selection of conserved blocks from multiple alignments for their use in phylogenetic analysis. Mol. Biol. Evol. 17, 540-552 (2000)

108. Guindon, S. et al. New algorithms and methods to estimate maximumlikelihood phylogenies: assessing the performance of PhyML 3.0. Syst. Biol. 59, 307-321 (2010)

109. Rambaut, A. FigTree, a graphical viewer of phylogenetic trees and as a program for producing publication-ready figures. http://tree.bio.ed.ac.uk/ software/figtree/ (2006)

110. Langmead, B. \& Salzberg, S. L. Fast gapped-read alignment with Bowtie 2. Nat. Methods 9, 357-359 (2012).

111. Barrero-Canosa, J. \& Moraru, C. Linking microbes to their genes at single cell level with direct-geneFISH. In: An Overview of FISH Concepts and Protocols for Microbial Cells (eds Almeida, C. \& Azevedo, N.). (Springer Nature, 2020).

112. Barrero-Canosa, J., Moraru, C., Zeugner, L., Fuchs, B. M. \& Amann, R. DirectgeneFISH: a simplified protocol for the simultaneous detection and quantification of genes and rRNA in microorganisms. Environ. Microbiol. 19, 70-82 (2017)

113. Perras, A. K. et al. S-layers at second glance? Altiarchaeal grappling hooks (hami) resemble archaeal S-layer proteins in structure and sequence. Front. Microbiol. 6, 543 (2015).

114. Wallner, G., Amann, R. \& Beisker, W. Optimizing fluorescent in situ hybridization with rRNA-targeted oligonucleotide probes for flow cytometric identification of microorganisms. Cytometry 14, 136-143 (1993).

115. Moissl, C., Rudolph, C., Rachel, R., Koch, M. \& Huber, R. In situ growth of the novel SM1 euryarchaeon from a string-of-pearls-like microbial community in its cold biotope, its physical separation and insights into its structure and physiology. Arch. Microbiol. 180, 211-217 (2003).

116. Flechsler, J. et al. 2D and 3D immunogold localization on (epoxy) ultrathin sections with and without osmium tetroxide. Microsc. Res. Tech. 83, 691-705 (2020).

117. Schlitzer, R. Data Analysis and Visualization with Ocean Data View, CMOS Bulletin SCMO. 43, 9-13 (2015).

\section{Acknowledgements}

This work received funding by the Alfred P. Sloan foundation (grant number G-20179955), the Ministry of Culture and Science of North Rhine-Westphalia (Nachwuchsgruppe "Dr. Alexander Probst"), and the NOVAC project of the German Science Foundation (grant number DFG PR1603/2-1). The authors acknowledge sampling logistics provided by the University of Regensburg, i.e., by Harald Huber and Sebastien Ferreira-Cerca, and sequencing of MSI metagenomes within the Census of Deep Life Sequencing call 2018, phase 14 project "Development of novel archaeal viruses and the corresponding CRISPR arrays of a highly abundant carbon fixer in Earth's crust". The authors highly appreciate the technical assistance of Jannis Becker for protein annotations of viral scaffolds, Lea Griesdorn for assistance with data submission, Sabrina Eisfeld for laboratory maintenance, and Ken Dreger for server administration and maintenance. The work conducted by the U.S. Department of Energy Joint Genome Institute, a DOE Office of Science User Facility, is supported under Contract No. DE-AC02-05CH11231. Furthermore, the authors are also grateful to the Imaging Center Essen (IMCES) under the direction of Matthias Gunzer for access to an inverted epifluorescence microscope with structured illumination and for the respective training carried out by Alexandra Brenzel.

\section{Author contributions}

J.R., V.T., P.A.F.-G., I.M. and A.J.P. performed the sampling; J.R., S.P.E., T.L.V.B., A.J.P., F.S., T.W. and A.J.P. carried out metagenomic analysis; J.R., V.T., I.M., A.K., C.M. and A.J.P. performed virusFISH or related analysis; V.T., I.M. and A.K. performed SIM and TEM microscopy; J.R., V.T. and A.J.P. wrote the manuscript; all authors contributed to editing and proofreading of the manuscript; and A.J.P. conceptualized the study.

\section{Funding}

Open Access funding enabled and organized by Projekt DEAL.

\section{Competing interests}

The author declares no competing interests. 


\section{Additional information}

Supplementary information The online version contains supplementary material available at https://doi.org/10.1038/s41467-021-24803-4.

Correspondence and requests for materials should be addressed to A.J.P.

Peer review information Nature Communications thanks Eugene Koonin, Mart Krupovic, and the other, anonymous, reviewer(s) for their contribution to the peer review of this work.

Reprints and permission information is available at http://www.nature.com/reprints

Publisher's note Springer Nature remains neutral with regard to jurisdictional claims in published maps and institutional affiliations. (c) (i) Open Access This article is licensed under a Creative Commons Attribution 4.0 International License, which permits use, sharing, adaptation, distribution and reproduction in any medium or format, as long as you give appropriate credit to the original author(s) and the source, provide a link to the Creative Commons license, and indicate if changes were made. The images or other third party material in this article are included in the article's Creative Commons license, unless indicated otherwise in a credit line to the material. If material is not included in the article's Creative Commons license and your intended use is not permitted by statutory regulation or exceeds the permitted use, you will need to obtain permission directly from the copyright holder. To view a copy of this license, visit http://creativecommons.org/ licenses/by/4.0/.

(C) The Author(s) 2021 